\title{
Frequency feedback for two-photon interference from separate quantum dots
}

\author{
Michael Zopf, ${ }^{1, *}$ Tobias Macha,,${ }^{2, *}{ }^{*}$ Robert Keil, ${ }^{1}$ Eduardo Uruñuela, ${ }^{2}$ Yan Chen, ${ }^{1}$ Wolfgang Alt, ${ }^{2}$ Lothar Ratschbacher, ${ }^{2}$ \\ Fei Ding, ${ }^{1,3, \ddagger}$ Dieter Meschede, ${ }^{2}$ and Oliver G. Schmidt ${ }^{1,4}$ \\ ${ }^{1}$ Institute for Integrative Nanosciences, Leibniz IFW Dresden, Helmholtzstraße 20, 01069 Dresden, Germany \\ ${ }^{2}$ Institut für Angewandte Physik, Universität Bonn, Wegelerstraße 8, 53115 Bonn, Germany \\ ${ }^{3}$ Institut für Festkörperphysik, Leibniz Universität Hannover, Appelstraße 2, 30167 Hannover, Germany \\ ${ }^{4}$ Material Systems for Nanoelectronics, Technische Universität Chemnitz, 09107 Chemnitz, Germany
}

(Received 22 December 2017; revised manuscript received 21 September 2018; published 12 October 2018)

\begin{abstract}
We employ active feedback to stabilize the frequency of single photons emitted by two separate quantum dots to an atomic standard. The transmission of a rubidium-based Faraday filter serves as the error signal for frequency stabilization. We achieve a residual frequency deviation of $<30 \mathrm{MHz}$, which is less than $1.5 \%$ of the quantum dot linewidth. Long-term stability is demonstrated by Hong-Ou-Mandel interference between photons from the two quantum dots. Their internal dephasing limits the expected visibility to $V=40 \%$. We observe $V_{\text {lock }}=(41 \pm 5) \%$ for frequency-stabilized dots as opposed to $V_{\text {free }}=(31 \pm 7) \%$ for free-running emission. Our technique reaches the maximally expected visibility for the given system and therefore facilitates quantum networks with indistinguishable photons from distributed sources.
\end{abstract}

DOI: 10.1103/PhysRevB.98.161302

Semiconductor quantum dots (QDs) are promising building blocks for photonic quantum computing [1], quantum communication [2,3], and applications in distributed quantum networks [4]. With recent efforts, not only are QDs able to emit high-quality single photons [5-7] and entangled photon pairs [8-13], they also match optical transitions in neutral atoms [14-16]. These constitute important elements in envisioned quantum repeaters and "quantum hybrid systems" $[17,18]$.

Interfacing the two platforms requires a stable emission frequency of the QD, which is sensitive to several external perturbations, including temperature $[19,20]$ as well as electric [21-23], magnetic [9,24], and strain fields [25-27]. While these phenomena lead to spectral wandering of the QD emission over long timescales, they simultaneously provide means to fine-tune and match the emission frequencies using active frequency feedback [28-30].

We simultaneously stabilize the emission frequency of two separate QDs via strain tuning of the host substrates [25,31]. For this purpose, a rubidium-based Faraday filter serves as an absolute frequency standard for distant nodes and acts as a tunable frequency discriminator at atomic transitions of rubidium, a prominent quantum memory candidate [32]. Our efficient feedback scheme, for which only a weak photon flux is necessary, along with the implementation of a common and reproducible standard, paves the way towards quantum networks with distributed, indistinguishable solid-state emitters.

In the following, we introduce the experimental setup and characterize the spectral quality of the QD emission,

\footnotetext{
${ }^{*}$ These authors contributed equally to this work.

†macha@iap.uni-bonn.de

${ }^{\ddagger}$ f.ding@fkp.uni-hannover.de
}

the frequency discriminator, and the feedback technique. As a benchmark, we show an improved long-term two-photon interference (TPI) visibility of the frequency-stabilized QDs in a Hong-Ou-Mandel experiment [19,33-35].

The GaAs/AlGaAs QDs were grown by solid-source molecular beam epitaxy and in situ $\mathrm{Al}$ droplet etching [14] and emit close to the rubidium $D_{1}$ transitions. Several QDcontaining nanomembranes are obtained using wet chemical etching and are bonded to a piezoelectric actuator $(0.3-\mathrm{mm}$ PMN-PT) via a flip-chip transfer process [36]. Precise emission wavelength control is then achieved by applying a voltage to the actuator.

The QD samples (QD1 and 2) are placed in two separate $\mathrm{He}$ cryostats at $4 \mathrm{~K}$ (see Fig. 1). A Ti:sapphire laser with 3 ps pulse length and $76 \mathrm{MHz}$ repetition rate is fed through a grating-based pulse-shaping setup for spectral narrowing. The pulses are then used to excite both QDs to the biexciton state (XX) in a resonant two-photon $\pi$-pulse condition [37]. The fine-structure splitting of the exciton state (X) leads to two cross-polarized XX decay channels, of which only one is selected by polarization filtering. The respective emission spectra are shown in Fig. 2(a).

Each QD emission is coupled into a single-mode fiber, delivering a photon rate of $R_{\mathrm{QD}} \approx 30 \mathrm{kcps}$. One part of each single-photon stream is sent to the TPI setup. It consists of a 50:50 nonpolarizing beam splitter, followed by monochromators for further background suppression and single-photon counting modules (SPCMs) in each output arm.

The emitted photons exhibit a Lorentzian emission profile with linewidths of $\theta^{(\mathrm{QD} 1)}=(2.08 \pm 0.01) \mathrm{GHz}$ and $\theta^{(\mathrm{QD} 2)}=$ $(2.59 \pm 0.08) \mathrm{GHz}$. In order to estimate the ideally expected TPI visibility, the lifetimes $T_{1}$ of the XX state and the coherence times $T_{2}$ of the respective photons are determined for both QDs. The derived photon indistinguishabilities 


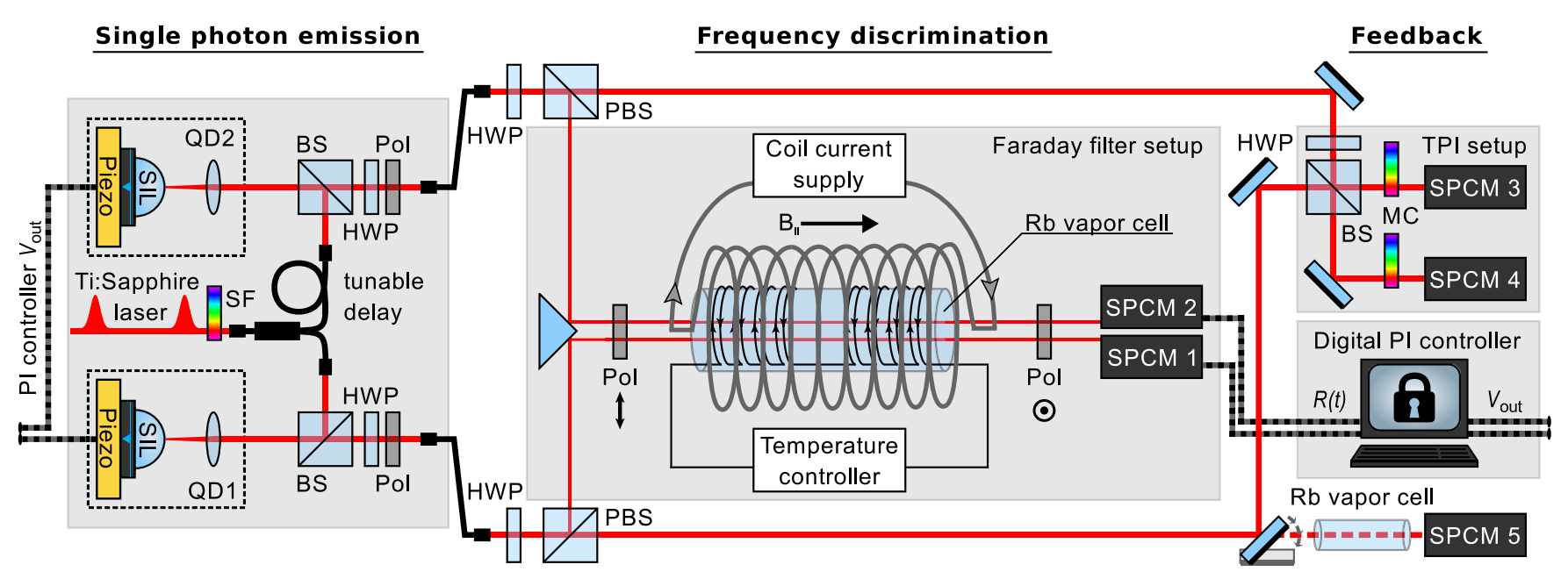

FIG. 1. Experimental setup for two-photon interference between separate, frequency-stabilized quantum dots (QDs). A pulsed Ti:sapphire laser resonantly excites the biexciton (XX) state of QD1 and QD2, positioned in separate cryostats at 4 K. Grating-based spectral filtering (SF) is applied to reduce the laser linewidth. A fiber-integrated, tunable delay adjusts the excitation to match arrival times of XX photons at the subsequent two-photon interference (TPI) setup. Both QDs are mounted on piezoelectric actuators for strain-induced emission frequency control. In each setup, the XX photons are collected using a confocal microscope, with the addition of a solid immersion lens (SIL) for enhanced extraction and a half-wave plate (HWP) and polarizer (Pol) for polarization filtering. A fraction of the signal is branched off by a HWP and a polarizing beam splitter (PBS) and sent through a Faraday filter setup. It consists of a heated, natural-abundance rubidium vapor cell in a longitudinal magnetic field, enclosed by two crossed polarizers. A coil current supply and a temperature controller enable tuning of the filter transmission features (see Fig. 2). The transmitted XX photons are detected by single-photon counting modules (SPCMs) 1 and 2 as signal inputs $R(t)$ for two digital proportional-integral (PI) controllers. Feedback voltages $V_{\text {out }}$ are generated and applied to the piezoelectric actuators for QD frequency stabilization. An additional Rb vapor cell in the signal arm of QD1 permits characterization of frequency drifts independent of the Faraday filter. The remaining XX photon streams are sent to the TPI setup, consisting of a beam splitter (BS), monochromators (MCs), and SPCMs 3 and 4. A HWP in one input arm is used to set the photon (in)distinguishability with respect to the polarization state.

$I=T_{2} / 2 T_{1}[38]$ are $I^{(\mathrm{QD} 1)}=(49.4 \pm 0.5) \%$ and $I^{(\mathrm{QD} 2)}=$ $(32.9 \pm 1.1) \%$. Interfering photons with identical frequencies from the two separate dots will therefore result in a maximum achievable visibility of $V=40 \%$ (see Supplemental Material [39]).

This expected visibility is comparable to other QD-based TPI experiments [19,35,40]. In most cases, internal dephasing processes in the QDs limit the photon indistinguishability. However, it has been shown that in principle visibilities as high as $98 \%$ can be obtained, e.g., by using charge-tunable QD devices [34]. For quantum repeater applications, photon indistinguishabilities of $99 \%$ are ultimately required [41]. As we will present in the following, the maximum visibilitytogether with tunability to an atomic transition-is only accessible using efficient frequency feedback.

The signal for frequency discrimination is provided by the Faraday effect. As depicted in Fig. 1, parts of the photon streams are directed to a rubidium-based Faraday anomalous dispersion optical filter (FADOF) setup [42,43]. Therefore off-resonant background signals are efficiently suppressed, while on-resonance photons are transmitted and detected by SPCMs. The expected transmission $\mathcal{T}_{\mathrm{QD}}$ is given by a convolution of a narrow-band, weak laser transmission $\mathcal{T}_{\mathrm{L}}$ with the spectral emission profile $f(v)$ of the $\mathrm{QD}: \mathcal{T}_{\mathrm{QD}}(v)=$ $\left(\mathcal{T}_{\mathrm{L}} * f\right)(v)$. Figure $2(\mathrm{~b})$ shows the expected $\mathcal{T}_{\mathrm{QD} 2}$ together with the measured frequency-tuned transmission of QD2. For a transmission peak close to the desired set frequency $v_{\text {set }}$, the slope around $v_{\text {set }}$ serves as the error signal for frequency stabilization. Changes in frequency are directly translated to a variation of the FADOF transmission. The latter also depends on both temperature $T$ and axially applied magnetic field $B_{\|}$[44], which provides a possibility to shift the transmission peak, as demonstrated in Fig. 2(c). Therefore, a desired frequency near an atomic hyperfine transition of rubidium can be addressed, in our case at the $D_{1}$ line $(795 \mathrm{~nm}$, see Supplemental Material [39]). Simultaneously, the width of the transmission peak can be adjusted to match the linewidth of the QD.

The SPCM photon detection rate $R_{\text {set }}=R\left(v_{\text {set }}\right)$ serves as the reference for frequency feedback. The rate $R$ of photon events at the SPCM can be written as

$$
R(t)=R[v(t)] \equiv \mathcal{T}_{\mathrm{QD}}[v(t)] \cdot R_{\mathrm{QD}},
$$

which depends on the time-varying center frequency $v(t)$ of the QD's spectral emission profile. By inverting Eq. (1), the instantaneous frequency deviation from the set point $\Delta v(t) \equiv$ $v(t)-v_{\text {set }}$ can be determined, using the observed detection rate $R(t)$. In practice, deviations from the set point are kept small by the feedback loop and the linearized relation

$$
\Delta v \approx \frac{1}{\left.\frac{d R}{d v}\right|_{\nu_{\mathrm{set}}}} \Delta R
$$

with $\Delta R=R(t)-R_{\text {set }}$ provides a good approximation.

In order to obtain an error signal for feedback, a simple, empirical algorithm is implemented to estimate the underlying scattering rate at any point in time. Motivated by the fact that photon events that lie further in the past convey less information and should thus be given lower weight with time, 
Single photon emission

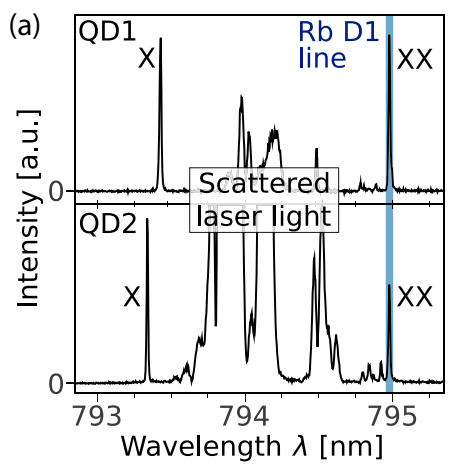

Frequency discrimination

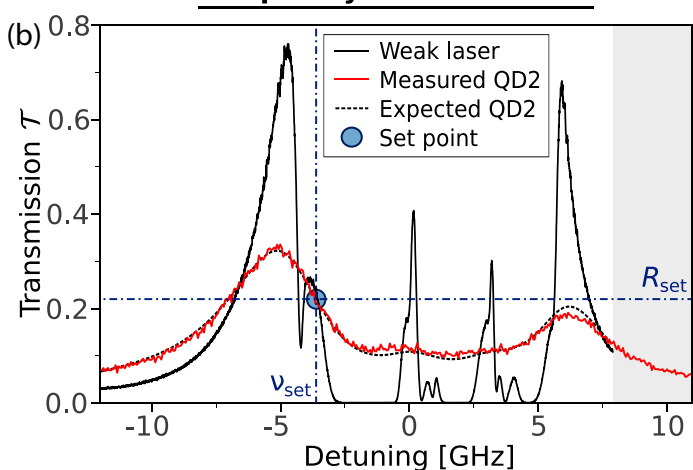

Feedback

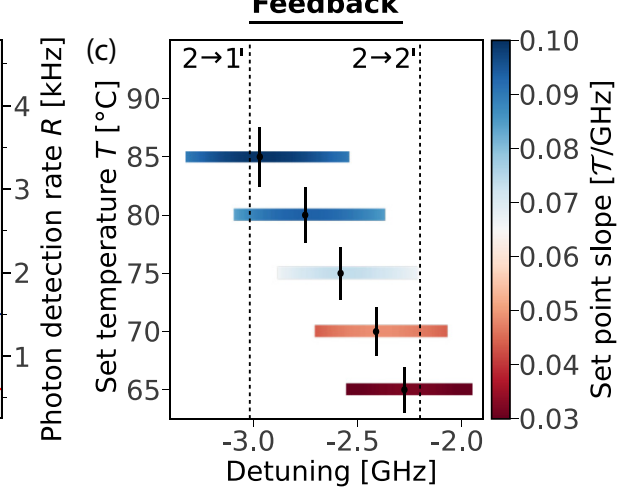

FIG. 2. (a) Emission spectra of two separate GaAs/AlGaAs QDs, whose emission frequencies are tuned in resonance with the Rb $D_{1}$ transition using piezo-induced strain fields. A two-photon excitation scheme resonantly addresses the biexciton state, which decays via the exciton state $(\mathrm{X})$ by emitting two consecutive photons (XX and $\mathrm{X}$ ). The scattered laser is partially suppressed using notch filters. (b) Faraday filter transmission spectra measured with a weak, narrow-band laser (solid black line) and with the frequency-tuned QD2 (red line). A convolution of the laser transmission with the spectral emission profile $f(v)$ of QD2 (dashed black line) is used to model the QD transmission. The detuning is given with respect to the weighted line center of the rubidium $D_{1}$ transitions. The set point for the TPI measurement (Fig. 3) with the reference photon rate $R_{\text {set }}=R\left(v_{\text {set }}\right)$ is highlighted. Only relatively weak photon streams (here, 6000 photons per second) are required to stabilize the frequency. The vapor cell was stabilized to a temperature of $85^{\circ} \mathrm{C}$ and the magnetic field was set to $40 \mathrm{mT}$. (c) Rubidium hyperfine transitions $F \rightarrow F^{\prime}$ are addressed by coarse adjustments of the set temperature $T$ and fine-tuning of the magnetic field $B_{\|}$. For different temperatures and $B_{\|}=29.5 \mathrm{mT}$ the maximum set point slopes are marked, along with the windows over which the slope decreases by less than $10 \%$. Stabilizing the frequency to other atomic resonances is discussed in the Supplemental Material [39].

an exponential smoothing filter is chosen to estimate the count rate $R(t)$. The digital implementation is similar to a first-order low-pass filter and described by the pseudocode

$$
R_{\text {estimate }, n+1}=R_{\text {estimate }, n} \cdot d+\mathcal{B} \cdot i,
$$

where

$$
\mathcal{B}= \begin{cases}1, & \text { if a photon arrived } \\ 0, & \text { else }\end{cases}
$$

with the decrement $d=e^{-\tau_{\text {cycle }} / \tau_{\text {filter }}}$ and the increment $i=$ $(1-d) / \tau_{\text {cycle }}$. Here, $\tau_{\text {cycle }}$ and $\tau_{\text {filter }}$ denote the cycle time of the digital loop and the chosen integration time of the filter, respectively. Instead of using a discrete averaging window [28], our algorithm represents an infinite impulse response filter and thus features a smooth frequency response.

There are two important aspects for rate-based frequency estimations: The first one is the correct detection of variations in the scattering rate $R(t)$ from the stochastic train of photon detection events observed by the SPCM. We measure the freerunning QD frequency-noise power spectral densities [45] on the rate $R(t)$ to determine the frequency at which the QD $1 / f$ noise is exceeded by detection shot noise. Then the feedback bandwidth of the control system is set to a frequency well below (see Supplemental Material [39]).

The second aspect is the distinction between rate variations due to frequency drifts and due to intensity changes in the QD emission. The latter could be compensated by adjusting the rate $R_{\text {set }}$ with respect to a rate measurement before the Faraday filter. Another possibility is creating a dispersive error signal by taking the difference of the orthogonal circular components of the filter transmission [42]. In our experiment, QD intensity fluctuations due to sample drifts are taken into account by selecting data windows in which the count rate after the TPI setup is stable.
The rate estimation algorithm as well as a subsequent standard digital proportional-integral (PI) controller are implemented on a field programmable gate array (FPGA) [46] using LABVIEW. The generated correction signal is sent to the strain-tuning piezoelectric actuator beneath the QD via a high-voltage amplifier.

Due to piezo creep, a certain set voltage on the piezoelectric actuator will not result in a constant strain in the QD membrane. The strain will slightly change over time and therefore result in a frequency drift, which is compensated by the implemented stabilization. For locking the QD emission frequencies, small count rates of only $R_{\text {set,QD1 }}=3600 \mathrm{cps}$ and $R_{\mathrm{set}, \mathrm{QD} 2}=1500 \mathrm{cps}$ are used. At the set point of $R_{\mathrm{set}, \mathrm{QD} 2}$, depicted in Fig. 2(b), the feedback bandwidth has to be limited to around $30 \mathrm{mHz}$ by adjusting the PI parameters (see Supplemental Material [39]). Figure 3(a) shows the frequency drift of QD1 for the frequency-locked and free-running case. It is determined by measuring the photon transmission through a separate, heated rubidium vapor cell, which constitutes an out-of-loop measurement of the frequency drift. Frequency stabilization leads to a constant frequency within a deviation of $<30 \mathrm{MHz}$ [47], which is less than $1.5 \%$ of the linewidths of the QDs $(\geqslant 2 \mathrm{GHz})$. In the free-running case, the frequency detuning $\Delta v(t)$ increases over time, following a logarithmic law known for the displacement change due to piezo creep [48],

$$
\Delta v(t)=\Delta v_{0}\left[1+\alpha \log _{10}\left(t-t_{0}\right)\right]
$$

Here, $\Delta v_{0}$ denotes the frequency detuning 1 min after a certain voltage is applied to the piezo at a time $t_{0}$, and $\alpha$ describes the rate of the piezo creep, which depends on the applied voltage and the piezo load. The displayed data in Fig. 3(a) is in good agreement with the model. The resulting 

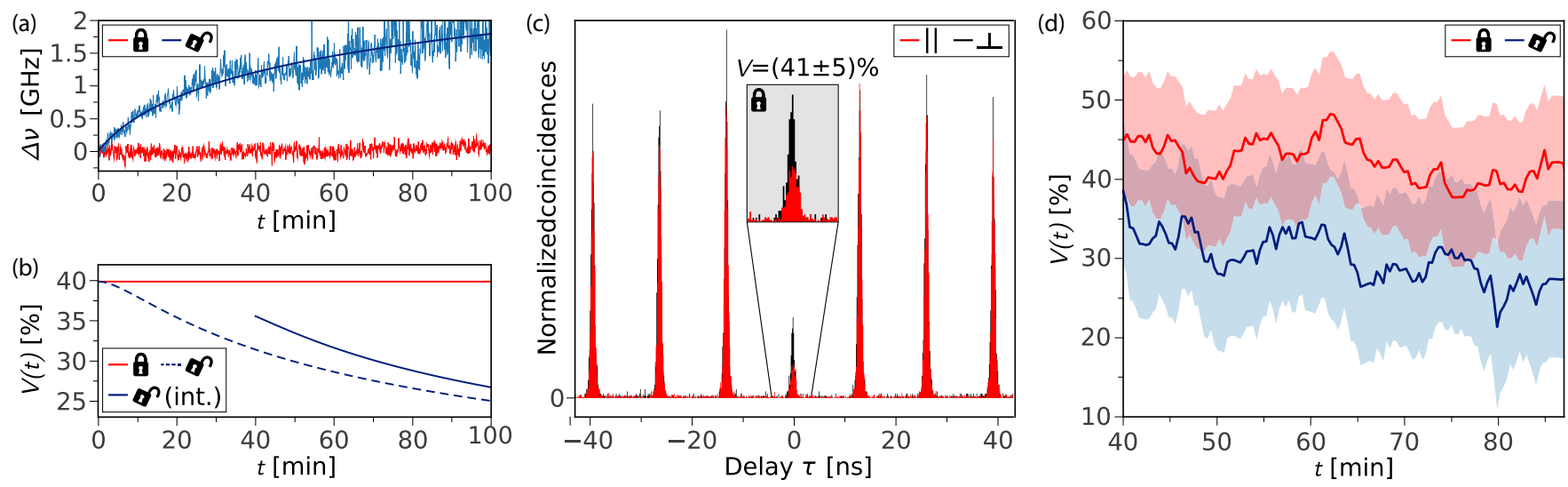

FIG. 3. (a) Relative emission-frequency drift over time for QD1 in frequency-locked and free-running state, obtained by an out-of-loop measurement of the transmission through a separate $\mathrm{Rb}$ vapor cell. In the stabilized state the frequency is kept constant within a deviation of less than $30 \mathrm{MHz}$, which is below $1.5 \%$ of the emission linewidth. The free-running state reveals a frequency drift due to piezo creep, which is fitted by a logarithmic function. (b) Theoretically expected evolution of the TPI visibility $V(t)$ considering the frequency drift shown in (a). We use the experimentally determined lifetimes $T_{1}$ and coherence times $T_{2}$ of the two separate QDs to model $V(t)$. While the visibility stays constant in the frequency-locked case (red line), for free-running QDs it drops from $V_{\text {free }}(t=0 \mathrm{~min})=40 \%$ to $V_{\text {free }}(t=100 \mathrm{~min})=25 \%$ (dashed line). The solid blue line represents the time-averaged visibility with a coincidence integration window of 40 min as used for the experiment in (d). (c) Two-photon interference measurement between two frequency-stabilized, separate QDs, showing the normalized coincidences vs the delay time $\tau$. The black curve corresponds to perpendicular polarizations of the photons impinging on the beam splitter. The red curve for parallel polarizations depicts a clear reduction of coincidences at $\tau \approx 0$. An interference visibility of $V_{\text {lock }}=(41 \pm 5) \%$ is obtained. A similar measurement of the visibility with free-running QDs (not shown) results in $V_{\text {free }}=(31 \pm 7) \%$. (d) Measurement of the interference visibility over time for both free-running and frequency-locked QDs. Each data point corresponds to the coincidences obtained for the previous 40 min. The shaded areas are the respective uncertainties based on Poisson counting statistics. At any measurement time the visibility is higher for frequency-locked QDs than for free-running QDs.

values for frequency drift over time are then used to calculate the theoretical TPI interference visibility for the locked and free-running QDs, taking the experimental parameters of the QD photons into account (see Supplemental Material [39]). Figure 3(b) shows the expected visibility over time, assuming a frequency drift between the two QD emission frequencies as observed in Fig. 3(a). Perfect frequency stability results in the maximum achievable visibility of $V=40 \%$, while for the measured piezo creep the theoretically expected visibility drops to $V=25 \%$ at $t=100 \mathrm{~min}$.

In order to experimentally verify an improved long-term visibility under frequency stabilization, we compare the TPI of photons from two separate QDs in the frequency-locked and free-running state. For stabilized QDs, Fig. 3(c) shows the normalized coincidences of photons in the two beam-splitter output ports versus the delay time $\tau$ between the recorded events. The polarization state between the interfering photons is controlled by a half-wave plate. The interference visibility $V$ is calculated by evaluating the peak areas $A_{\|}$for parallel and $A_{\perp}$ for perpendicular polarizations of photons impinging on the beam splitter at $\tau=0$,

$$
V=\frac{A_{\perp}-A_{\|}}{A_{\perp}} .
$$

A clear Hong-Ou-Mandel dip is observed, yielding an interference visibility of $V_{\text {lock }}=(41 \pm 5) \%$ after dark count correction of the SPCMs $\left(R_{\mathrm{dc}, \mathrm{SPCM} 3}=104 \mathrm{cps}, R_{\mathrm{dc}, \mathrm{SPCM} 4}=\right.$ $134 \mathrm{cps})$. The visibility agrees well with the expected value of $V=40 \%$ in Fig. 3(b). Afterwards, a measurement with free-running QDs is performed. The visibility in that case decreases to $V_{\text {free }}=(31 \pm 7) \%$, due to piezo creep and other emission frequency perturbations. For ideal quantum emitters the ratio of the peak at $\tau=0$ compared to the neighboring peaks equals 0.5 for perpendicular photon polarizations [6]. Here, a lower ratio is observed, which can be attributed to blinking of the QD emission [49]. This effect happens at submicrosecond timescales, to which the frequency stabilization is insensitive. To further compare the two cases of TPI with and without frequency feedback, the interference visibility is measured as a function of time, as shown in Fig. 3(d). Each respective data point corresponds to the coincidences obtained within the previous $40 \mathrm{~min}$. Hence, the integration window is gradually shifted through the total measurement time of $87 \mathrm{~min}$. The shaded areas display the respective uncertainties due to Poissonian counting statistics. In the locked and free-running case, both QDs were frequency matched at $t=0 \mathrm{~min}$. In the free-running case, frequency changes in the QD emission within the first integration window reduce the visibility already for the first data points [see Fig. 3(b)].

In conclusion, we have verified that active frequency feedback based only on measurements of the emitted single photons is an attractive solution to maintain long-term indistinguishability of photons from separate solid-state emitters. Stable two-photon interference from separate quantum dots is achieved by strain-mediated frequency stabilization. Frequency fluctuations are suppressed to a negligible fraction of the emission linewidth. The rubidium-based Faraday filter offers a common, absolute frequency reference for distant nodes in a quantum network. Furthermore, matching atomic 
transitions is desirable for atom-based quantum memories as potential elements in quantum repeaters. Low filter losses and an efficient rate-estimation algorithm ensure frequency stabilization while using only a small fraction of the photon flux.

The presented experiment can be extended to stabilize the emission of entangled photons for realizing a stable Bell state measurement in entanglement swapping schemes. In the case of QDs this means maintaining a low fine-structure splitting by separating emitted photons according to their polarization and using two orthogonal degrees of freedom for feedback, as available in anisotropic strain-tuning platforms [27].

We acknowledge funding by the BMBF (Q.com), the European Commission (HANAS), and the European Research Council (QD-NOMS). T.M. and E.U. thank the BCGS, and L.R. the Alexander von Humboldt Foundation. F.D. acknowledges support by IFW Excellence Program.
[1] J. L. O’Brien, Science 318, 1567 (2007).

[2] N. Gisin and R. Thew, Nat. Photonics 1, 165 (2007).

[3] H. de Riedmatten, I. Marcikic, J. A. W. van Houwelingen, W. Tittel, H. Zbinden, and N. Gisin, Phys. Rev. A 71, 050302(R) (2005).

[4] J. W. Pan, Z. B. Chen, C. Y. Lu, H. Weinfurter, A. Zeilinger, and M. Zukowski, Rev. Mod. Phys. 84, 777 (2012).

[5] P. Michler, A. Kiraz, C. Becher, W. V. Schoenfeld, P. M. Petroff, L. Zhang, E. Hu, and A. Imamoglu, Science 290, 2282 (2000).

[6] C. Santori, M. Pelton, G. Solomon, Y. Dale, and Y. Yamamoto, Phys. Rev. Lett. 86, 1502 (2001).

[7] Y.-M. He, Y. He, Y.-J. Wei, D. Wu, M. Atatüre, C. Schneider, S. Höfling, M. Kamp, C.-Y. Lu, and J.-W. Pan, Nat. Nanotechnol. 8, 213 (2013).

[8] O. Benson, C. Santori, M. Pelton, and Y. Yamamoto, Phys. Rev. Lett. 84, 2513 (2000).

[9] R. M. Stevenson, R. J. Young, P. Atkinson, K. Cooper, D. A. Ritchie, and A. J. Shields, Nature (London) 439, 179 (2006).

[10] N. Akopian, N. H. Lindner, E. Poem, Y. Berlatzky, J. Avron, D. Gershoni, B. D. Gerardot, and P. M. Petroff, Phys. Rev. Lett. 96, 130501 (2006).

[11] R. Hafenbrak, S. M. Ulrich, P. Michler, L. Wang, A. Rastelli, and O. G. Schmidt, New J. Phys. 9, 315 (2007).

[12] A. Muller, W. Fang, J. Lawall, and G. S. Solomon, Phys. Rev. Lett. 103, 217402 (2009).

[13] A. Dousse, J. Suffczyński, A. Beveratos, O. Krebs, A. Lemaître, I. Sagnes, J. Bloch, P. Voisin, and P. Senellart, Nature (London) 466, 217 (2010).

[14] R. Keil, M. Zopf, Y. Chen, B. Höfer, J. Zhang, F. Ding, and O. G. Schmidt, Nat. Commun. 8, 15501 (2017).

[15] P. Atkinson, E. Zallo, and O. G. Schmidt, J. Appl. Phys. 112, 054303 (2012).

[16] S. Kumar, R. Trotta, E. Zallo, J. D. Plumhof, P. Atkinson, A. Rastelli, and O. G. Schmidt, Appl. Phys. Lett. 99, 161118 (2011).

[17] M. T. Rakher, R. J. Warburton, and P. Treutlein, Phys. Rev. A 88, 053834 (2013).

[18] J. Wolters, G. Buser, A. Horsley, L. Béguin, A. Jöckel, J.-P. Jahn, R. J. Warburton, and P. Treutlein, Phys. Rev. Lett. 119, 060502 (2017).

[19] V. Giesz, S. L. Portalupi, T. Grange, C. Antón, L. De Santis, J. Demory, N. Somaschi, I. Sagnes, A. Lemaître, L. Lanco, A. Auffèves, and P. Senellart, Phys. Rev. B 92, 161302 (2015).

[20] X. Ding, Y. He, Z.-C. Duan, N. Gregersen, M.-C. Chen, S. Unsleber, S. Maier, C. Schneider, M. Kamp, S. Höfling, C.-Y. Lu, and J.-W. Pan, Phys. Rev. Lett. 116, 020401 (2016).
[21] A. J. Bennett, M. A. Pooley, R. M. Stevenson, M. B. Ward, R. B. Patel, A. B. de la Giroday, N. Sköld, I. Farrer, C. A. Nicoll, D. A. Ritchie, and A. J. Shields, Nat. Phys. 6, 947 (2010).

[22] M. Ghali, K. Ohtani, Y. Ohno, and H. Ohno, Nat. Commun. 3, 661 (2012)

[23] J. Zhang, E. Zallo, B. Höfer, Y. Chen, R. Keil, M. Zopf, S. Böttner, F. Ding, and O. G. Schmidt, Nano Lett. 17, 501 (2017).

[24] M. A. Pooley, A. J. Bennett, R. M. Stevenson, A. J. Shields, I. Farrer, and D. A. Ritchie, Phys. Rev. Appl. 1, 024002 (2014).

[25] F. Ding, R. Singh, J. D. Plumhof, T. Zander, V. Křápek, Y. H. Chen, M. Benyoucef, V. Zwiller, K. Dörr, G. Bester, A. Rastelli, and O. G. Schmidt, Phys. Rev. Lett. 104, 067405 (2010).

[26] J. Zhang, Y. Huo, A. Rastelli, M. Zopf, B. Höfer, Y. Chen, F. Ding, and O. G. Schmidt, Nano Lett. 15, 422 (2015).

[27] Y. Chen, J. Zhang, M. Zopf, K. Jung, Y. Zhang, R. Keil, F. Ding, and O. G. Schmidt, Nat. Commun. 7, 10387 (2016).

[28] M. Metcalfe, A. Muller, G. S. Solomon, and J. Lawall, J. Opt. Soc. Am. B 26, 2308 (2009).

[29] J. H. Prechtel, A. V. Kuhlmann, J. Houel, L. Greuter, A. Ludwig, D. Reuter, A. D. Wieck, and R. J. Warburton, Phys. Rev. X 3, 041006 (2013).

[30] N. Akopian, R. Trotta, E. Zallo, S. Kumar, P. Atkinson, A. Rastelli, O. G. Schmidt, and V. Zwiller, arXiv:1302.2005.

[31] R. Trotta, P. Atkinson, J. D. Plumhof, E. Zallo, R. O. Rezaev, S. Kumar, S. Baunack, J. R. Schröter, A. Rastelli, and O. G. Schmidt, Adv. Mater. 24, 2668 (2012).

[32] M. Körber, O. Morin, S. Langenfeld, A. Neuzner, S. Ritter, and G. Rempe, Nat. Photonics 12, 18 (2018).

[33] C. K. Hong, Z. Y. Ou, and L. Mandel, Phys. Rev. Lett. 59, 2044 (1987).

[34] R. B. Patel, A. J. Bennett, I. Farrer, C. A. Nicoll, D. A. Ritchie, and A. J. Shields, Nat. Photonics 4, 632 (2010).

[35] E. B. Flagg, A. Muller, S. V. Polyakov, A. Ling, A. Migdall, and G. S. Solomon, Phys. Rev. Lett. 104, 137401 (2010).

[36] Y. Zhang, Y. Chen, M. Mietschke, L. Zhang, F. Yuan, S. Abel, R. Hühne, K. Nielsch, J. Fompeyrine, F. Ding, and O. G. Schmidt, Nano Lett. 16, 5785 (2016).

[37] M. Müller, S. Bounouar, K. D. Jöns, M. Glässl, and P. Michler, Nat. Photonics 8, 224 (2014).

[38] J. Bylander, I. Robert-Philip, and I. Abram, Eur. Phys. J. D 22, 295 (2003).

[39] See Supplemental Material at http://link.aps.org/supplemental/ 10.1103/PhysRevB.98.161302 for details and supporting measurements.

[40] A. Thoma, P. Schnauber, J. Böhm, M. Gschrey, J.-H. Schulze, A. Strittmatter, S. Rodt, T. Heindel, and S. Reitzenstein, Appl. Phys. Lett. 110, 011104 (2017). 
[41] I. Aharonovich, D. Englund, and M. Toth, Nat. Photonics 10, 631 (2016).

[42] S. L. Portalupi, M. Widmann, C. Nawrath, M. Jetter, P. Michler, J. Wrachtrup, and I. Gerhardt, Nat. Commun. 7, 13632 (2016).

[43] J. A. Zielińska, F. A. Beduini, N. Godbout, and M. W. Mitchell, Opt. Lett. 37, 524 (2012).

[44] The software ELECSUS [50] is used to calibrate the conversion from coil current to magnetic field.

[45] A. V. Kuhlmann, J. Houel, A. Ludwig, L. Greuter, D. Reuter, A. D. Wieck, M. Poggio, and R. J. Warburton, Nat. Phys. 9, 570 (2013).
[46] National Instruments NI PXI-7842R card.

[47] Calculated using $\sqrt{\sigma_{N}^{2}-\bar{N}}$ to exclude the detection shot noise. $\bar{N}$ is the average count number for $\geqslant 0.5 \mathrm{~s}$ binning times and $\sigma_{N}$ is the corresponding standard deviation.

[48] H. Jung and D.-G. Gweon, Rev. Sci. Instrum. 71, 1896 (2000).

[49] K. D. Jöns, K. Stensson, M. Reindl, M. Swillo, Y. Huo, V. Zwiller, A. Rastelli, R. Trotta, and G. Björk, Phys. Rev. B 96, 075430 (2017).

[50] M. A. Zentile, J. Keaveney, L. Weller, D. J. Whiting, C. S. Adams, and I. G. Hughes, Comput. Phys. Commun. 189, 162 (2015). 